\title{
Analytical validation of whole exome and whole genome sequencing for clinical applications
}

\author{
Michael D Linderman ${ }^{1,2^{*}}$, Tracy Brandt², Lisa Edelmann², Omar Jabado ${ }^{1,2}$, Yumi Kasai ${ }^{1,2}$, Ruth Kornreich², \\ Milind Mahajan ${ }^{1,2}$, Hardik Shah ${ }^{1,2}$, Andrew Kasarskis ${ }^{1,2}$ and Eric E Schadt ${ }^{1,2}$
}

\begin{abstract}
Background: Whole exome and genome sequencing (WES/WGS) is now routinely offered as a clinical test by a growing number of laboratories. As part of the test design process each laboratory must determine the performance characteristics of the platform, test and informatics pipeline. This report documents one such characterization of WES/WGS.

Methods: Whole exome and whole genome sequencing was performed on multiple technical replicates of five reference samples using the Illumina HiSeq 2000/2500. The sequencing data was processed with a GATK-based genome analysis pipeline to evaluate: intra-run, inter-run, inter-mode, inter-machine and inter-library consistency, concordance with orthogonal technologies (microarray, Sanger) and sensitivity and accuracy relative to known variant sets.
\end{abstract}

Results: Concordance to high-density microarrays consistently exceeds 97\% (and typically exceeds 99\%) and concordance between sequencing replicates also exceeds $97 \%$, with no observable differences between different flow cells, runs, machines or modes. Sensitivity relative to high-density microarray variants exceeds $95 \%$. In a detailed study of a $129 \mathrm{~kb}$ region, sensitivity was lower with some validated single-base insertions and deletions "not called". Different variants are "not called" in each replicate: of all variants identified in WES data from the NA12878 reference sample $74 \%$ of indels and $89 \%$ of SNVs were called in all seven replicates, in NA12878 WGS $52 \%$ of indels and $88 \%$ of SNVs were called in all six replicates. Key sources of non-uniformity are variance in depth of coverage and artifactual variants resulting from repetitive regions and larger structural variants.

Conclusion: We report a comprehensive performance characterization of WES/WGS that will be relevant to offering laboratories, consumers of genome sequencing and others interested in the analytical validity of this technology.

\section{Background}

Whole exome and genome sequencing (WES/WGS) is now routinely offered as a clinical test by a growing number of laboratories. WES/WGS is implemented as a laboratory-developed test that must be fully validated by the offering laboratory prior to use. This validation effort "establishes the analytical performance for the clinical test system ... to confirm that the system is suitable for its intended use" [1], and in this context (next-generation sequencing) is focused on three inter-related aspects: platform, test-specific and informatics pipeline validation.

\footnotetext{
* Correspondence: michael.linderman@mssm. edu

${ }^{1}$ Icahn Institute for Genomics and Multiscale Biology, Icahn School of Medicine at Mount Sinai, New York, NY, USA

${ }^{2}$ Department of Genetics and Genomic Sciences, Icahn School of Medicine at Mount Sinai, New York, NY, USA
}

This report documents one such validation of WES/WGS for patients without a molecular diagnosis but suspected constitutional disease mutation(s), with attention to parameters that measure the reproducibility of the testing platform as well as the informatics pipeline. We performed a focused evaluation of the analytical performance characteristics of SNV and small indel (less than $50 \mathrm{bp}$ ) detection for a single workflow across multiple technical replicates. This study complements the comparisons of different sequencing technologies $[2,3]$, exome capture techniques $[4,5]$ and informatics pipelines [6,7] that have been reported previously.

The American College of Medical Genetics (ACMG) has developed clinical laboratory standards for NGS [8], which specifically address the unique challenges of WES/WGS. Since WES/WGS is not targeting specific diseases/genes, the validation effort is not focused on the specific sequence contexts and variant types associated 
with those diseases, but rather on developing and evaluating end-to-end metrics for high quality sequencing. Different expectations apply to WES/WGS than targeted panels or single gene testing. For instance, instead of "sequence-to-completion" for a specific region the goal is to sensitively and precisely call variants over the largest percentage of the target region possible, while also being able to determine and report which bases did not meet the minimum requirements for successful variant calling.

The variant calling error rate is determined by many parameters; setting a single minimum threshold for confident calls is challenging. We do not attempt to define a fixed set of quality filters that produce our desired sensitivity and specificity. Instead we use variant quality score recalibration (VQSR) $[9,10]$, a statistical technique for variant filtration that builds a model of "true" variants using multiple quality parameters and then applies that model to filter out likely false positive variants. With VQSR we set desired end-to-end sensitivity and determine the thresholds for specific quality parameters directly from the data itself. All performance characteristics were measured in the context of this filtering approach. VQSR can be combined with separate filters used to flag for additional investigation genomic regions with increased likelihood of missed/artifactual variants or genotypes with increased likelihood of incorrect zygosity.

The scale of WES/WGS makes it prohibitive to evaluate all variants with alternative technologies and the cost limits the number of samples that can be sequenced as part of the validation process. We sequenced multiple technical replicates of five reference samples in a scheme to extensively but efficiently test intra-run, inter-run, inter-machine and inter-mode reproducibility. We compared this data to a variety of reference callsets including SNP arrays, Sanger validation data from targeted NGS panels offered by our laboratory and publicly available variant calls to evaluate accuracy, sensitivity and specificity. Ongoing standardization efforts are continually producing additional and improved reference materials (RMs) and associated callsets [11]; in this report, for example, we use the first release of the "Genomes In a Bottle" (GIAB) variant callset [12]. The rapid evolution of sequencing technology, informatics tools and RMs make validation and optimization a continuous process in which these results represent a particular moment in time. The process we describe, however, is largely automated and readily adaptable to new informatics tools and data resources as they become available.

\section{Methods}

\section{Materials}

The samples listed in Table 1 were sequenced as multiple technical replicates according to the schematics
Table 1 Summary of samples used in validation experiments with known variants as reported by Coriell

\begin{tabular}{llll}
\hline Sample & Sex & Known variants & Note \\
\hline NA12878 & F & $\begin{array}{l}\text { Heterozygous CYP2C19 } \\
\text { C.681G > A (rs4244285) }\end{array}$ & \\
NA12891 & M & $\begin{array}{l}\text { Homozygous CYP2C19 } \\
\text { C.681G > A (rs4244285) }\end{array}$ & Father to NA12878 \\
NA12892 & F & N/A & Mother to NA12878 \\
NA10080 & M & Heterozygous PTEN c.781C > T & \\
NA18507 & M & N/A & \\
\hline
\end{tabular}

shown in Figures 1 and 2. The samples were chosen to include a trio (NA12878-NA12891-NA12892) with numerous reference callsets both public and internal, ethnic diversity (NA18507) and a sample (NA10080) with a known disease mutation and internal reference materials. The replicates were designed to test intra-run (same run of same machine), inter-run (different run of same machine), inter-library, inter-machine and intermode (between high-throughput and rapid run modes) reproducibility. Note that each pair-wise comparison between replicates may represent more than one of the above comparisons; tables of comparison type are included in the Additional file 1. Replicates are named by sample/run-machine-slot. DNA was derived from lymphoblastoid cell lines from the Coriell Institute for Medical Research.

Table 2 lists the reference materials used in this analysis.

\section{Sequencing and variant calling}

WES and WGS was performed on a HiSeq 2000/2500 (Illumina, San Diego, CA, USA) with a 100 base-pair (bp) paired-end protocol. WES samples were barcoded and pooled with up to three other samples prior to enrichment for exonic DNA with the Nimblegen SeqCap EZ Human Exome Library v3.0. The sequencing data is available via the SRA under BioProjects PRJNA241071 and PRJNA241062.

The genome analysis pipeline (GAP) is based on the 1000 Genomes Project (1000G) data analysis data pipeline and is composed from the widely used open source software projects bwa 0.7.5a [13], Picard 1.96 [14], GATK 2.7 [9,10], snpEff 3.0 [15], BEDTools 2.16.2 [16] and custom-developed software. Short-reads are aligned to a gender- and pseudo-autosomal region (PAR)-masked build of the hg19 human reference genome using bwa mem. The GAP implements the "GATK Best Practices" including indel realignment, de-duplication, and basequality score recalibration (BQSR).

Single nucleotide variants (SNVs) and indels were called jointly with the GATK HaplotypeCaller. Variant quality score recalibration (VQSR) was used to estimate 
the probability that a WES or WGS SNV is a true variant instead of an artifact and set the corresponding variant filter thresholds. VQSR was used for WGS but not WES indels, due to an insufficient number of variants to train the model; fixed filters were used instead. The PASS threshold for VQSR is set to capture of $99.5 \%$ known true-positives. We observed this threshold to offer a good compromise between precision and recall; the impact of changing this threshold can be observed in the results section in the context of the Genome in a Bottle (GIAB) reference material. Note that in choosing a threshold below $100 \%$ we set a corresponding minimum false negative rate.

The exome capture targets were expanded with 100 bp flanks for variant calling. Mean coverage and factions of bases at different coverage levels were calculated with the un-flanked intervals; the callable coverage of RefSeq coding exons was calculated with the flanked intervals.

\section{Array genotyping}

Array genotyping was performed on 750 nanograms of DNA with the Human Omni2.5 BeadChip (Illumina, San Diego, CA, USA). The hybridized arrays were scanned using the HiScan system (Illumina, San Diego, CA, USA) and the genotypes called utilizing the software
GenomeStudio v1.0 Genotyping Module (Illumina, San Diego, CA, USA).

\section{Validation statistics}

Genotype concordance (concordance) and the related metrics, non-reference sensitivity (NRS), non-reference genotype concordance (NRC) and precision are computed as the ratio of solid red elements to blue outline elements shown in Figure 3 (where " $\mathrm{B}$ " is the non-reference allele). Non-PASSing variants are treated as "no-calls" (./.) and multi-allelic sites are decomposed into their component bi-allelic variants. Callsets are joined using strict variant equality (position, reference and alternate alleles); thus different representations of the same complex variant will not be recognized as concordant. However, wherever relevant (ASD panel callset) all variants have been similarly leftaligned to reduce this effect. And we note that the GIAB callset was regularized with the same variant caller (GATK Haplotype Caller). Where relevant, and unless otherwise specified, all metrics are computed with respect to the target intervals associated with the callset e.g. the target intervals for any gene panel and the "high-confidence" intervals supplied alongside the GIAB callset.

All metrics are sensitive to the choice of variants in the truth and test sets, especially the concordance

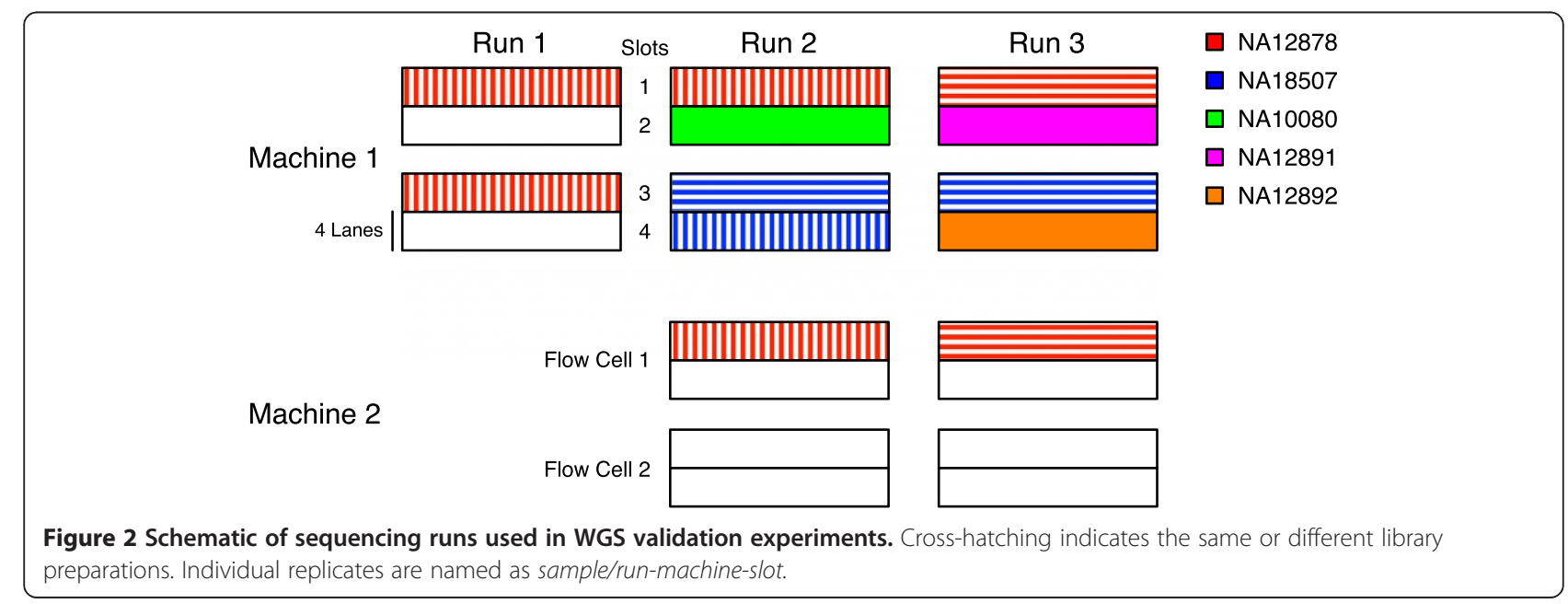


Table 2 Description of reference callsets used in analysis

\begin{tabular}{ll}
\hline Dataset & Notes (version, target intervals, etc.) \\
\hline $\begin{array}{l}\text { In-house Omni 2.5 } \\
\text { Microarray }\end{array}$ & Described in methods section \\
1000G Omni 2.5 & As distributed in GATK Resource Bundle version 2.3 \\
$\begin{array}{l}\text { Microarray } \\
\text { Hapmap }\end{array}$ & $\begin{array}{l}\text { Version 3.3. As distributed in GATK Resource Bundle } \\
\text { version 2.3 }\end{array}$ \\
$\begin{array}{l}\text { Genome in a } \\
\text { Bottle }\end{array}$ & $\begin{array}{l}\text { "hersion 2.17, dated Oct. 17 2013. Most restrictive } \\
\text { repeats, segmental duplications, decoys, STRs, and }\end{array}$ \\
known CNVs. \\
$\begin{array}{l}\text { Autism(ASD) } \\
\text { Panel }\end{array}$ & $\begin{array}{l}\text { 129 kilobase targeted clinical sequencing panel of } \\
\text { Indels are left aligned. }\end{array}$ \\
\hline
\end{tabular}

metric, which is determined by the intersection of the "test" and "truth" datasets. Including many homozygous reference genotypes, which can be easier to call, can bias the concordance metric. The GAP only reports variants from the reference, minimizing the biasing effect of homozygous reference calls. Similarly, restricting the variants in the test set to only very high quality variants will also bias the concordance statistic, as would restricting the variants included in the truth set to those readily called in NGS data. All NGS replicates use the filtering scheme described previously, and any filters applied to the reference materials are specified.

Although the SNP microarrays and other callsets are treated as "truth", they are not error free. The error rate for the BeadArray technology is estimated at 0.3\% [17], although the actual error rate will be a function of the QC strategy employed and as the data shown here suggests, is actually higher, than that estimate in this context. There is no "truth" callset when comparing two WES or WGS callsets that should be identical. In these cases we conduct the same concordance analysis twice, treating each callset alternately as both "truth" and "test". By definition the concordance is symmetric, but differences in NRS, NRC and Precision will be observed.

\section{Experiments}

Table 3 summarizes the different validation experiments performed. Metrics are computed as described previously.
The WES and WGS replicates used in different experiments are listed in Table 4, with all replicates used for concordance testing against alternate technologies and curated variant sets.

\section{Results and discussion}

Sequencing statistic

Tables 5 and 6 list the coverage statistics for the different WES and WGS replicates, respectively. The percentage of RefSeq coding bases considered confidently callable represents an estimate of the portion of those bases over which we could confidently call variants. Confidence is determined by: a minimum of 20 -fold coverage and no more than 10\% MAPQ0 (ambiguously mapped) reads. This estimate is intended to be conservative (the coverage threshold is set at the upper end of the range suggested in the guidelines [8]) however, we may still fail to call variants in regions that are considered "confidently callable".

\section{Microarrays}

Figure 4 shows the concordance, NRS and NRC relative to three different SNP microarray genotypes (the "In-house Omni 2.5 Microarray", "1000G Omni 2.5 Microarray" and "Hapmap" reference callsets). The concordance exceeds $97 \%$ (98.5\% for more filtered $1000 \mathrm{G}$ array), with the NRS exceeding 92.5\% (95\% excluding HapMap). These results are consistent with the concordance rates listed in the guidelines [8]. The observed NRS is lower than the sensitivity set in VQSR (99.5\%), even though the same datasets (1000G Omni 2.5 and Hapmap 3.3 genotypes) are used in that training process, showing the impact of insufficient coverage and various error processes, e.g. low complexity and structural variants, on end-to-end sensitivity. For example, more missed variants are located on the hyper-variable MHC region of chromosome 6 , than any other chromosome region. The NRS increases 2-3\% if we restrict the concordance analysis to those variants in regions identified as callable per the requirements specified above.

The stratification in NRS and NRC reflects the different technologies and QC procedures of the array data sources. More filtering was applied to the 1000G Omni 2.5 genotypes

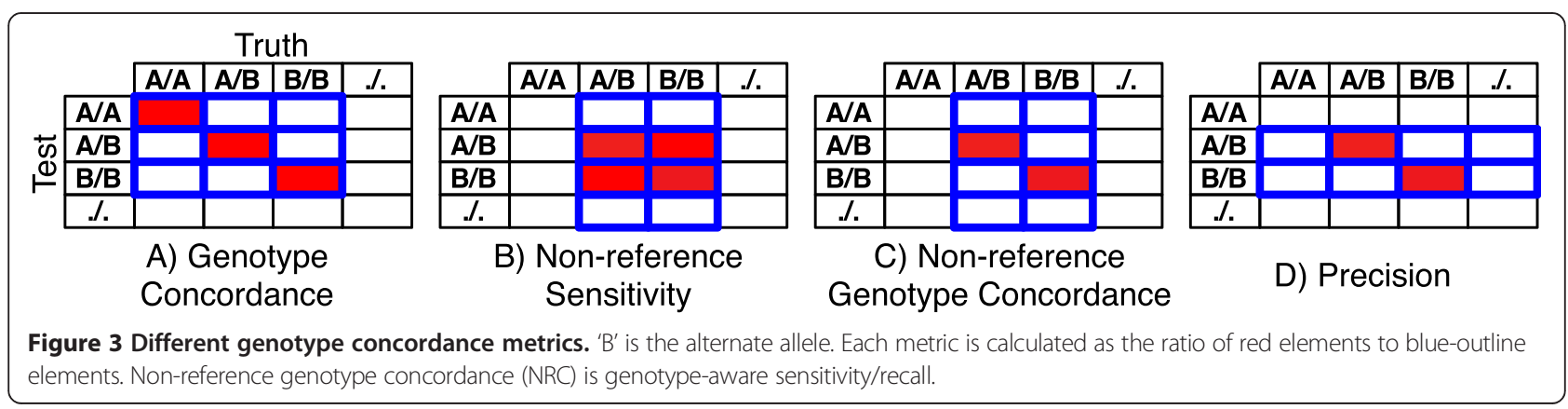




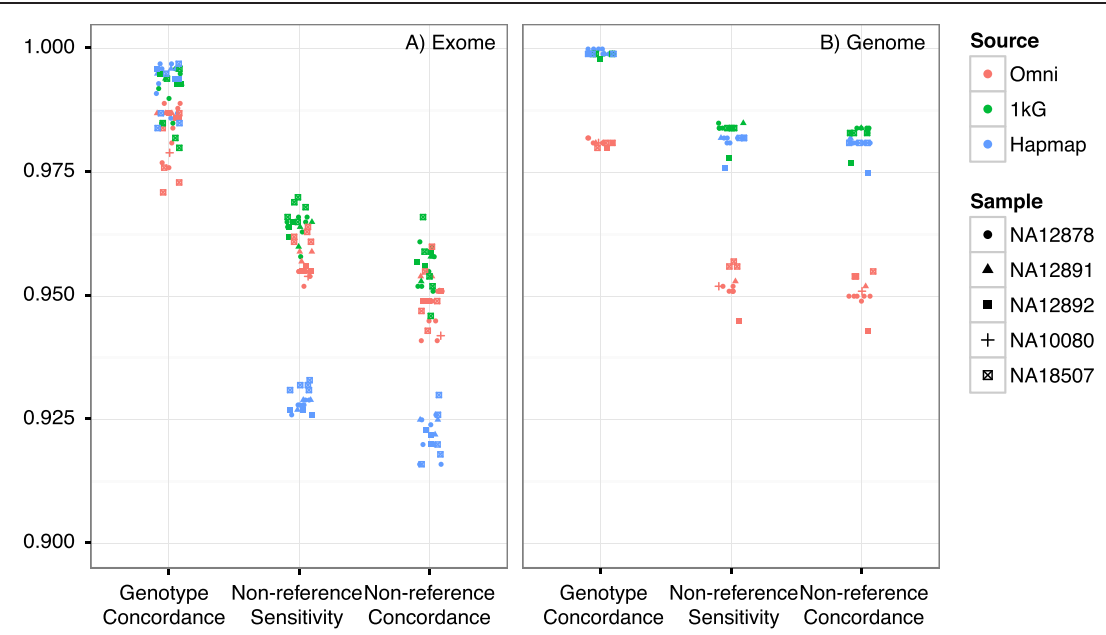

Figure 4 WES (A) and WGS (B) genotype concordance (concordance), non-reference sensitivity (NRS) and non-reference concordance (NRC) relative to three different SNP microarray genotypes: 1) an Illumina Omni2.5 genotype performed in-house, 2) an Omni2.5 genotype performed as part of the 1000 Genomes project, and 3) Hapmap 3.3 genotype. Not all genotypes are available for all samples. Only those SNPs within the exome capture targets are considered for WES concordance. The GAP does not report non-variant sites, so homozygous reference calls are not considered in the concordance evaluation.

by its providers [18], such as flagging SNVs within 20 bp of an indel, than the Omni 2.5 genotyping performed in-house, which has no specific QC filters applied. We expect higher NRS and NRC relative to the $1000 \mathrm{G}$ arrays as a result. Unlike the Omni arrays, the HapMap dataset was lifted to hg19 from hg18/GRCh36. The resulting mapping errors could introduce artifactual variants that bias sensitivity.

\section{Genome in a bottle}

The mean concordance, NRS and NRC relative to the GIAB variant callset (with full range in brackets), restricted to variants within the GIAB high-confidence regions, are 99.0\% [98.2,99.6], 96.1\% [95.4,96.5] and 95.1\% [94.5,95.8], respectively, for WES and 99.7\% [99.6,99.7], 94.2\%
[93.0,95.4] and 93.9\% [92.7,95.1], respectively, for WGS. All metrics are improved compared to using the microarrays as the truth callset, as would be expected. The GIAB callset is produced from similar NGS datasets, using a similar analysis pipeline, and the analysis was restricted to the GIAB high-confidence regions. If we further restrict to those variants in regions identified in each replicate as callable (and thus eliminate low coverage regions in each sample), the mean NRS increases to $98.9 \%$ and $95.0 \%$ for WES and WGS respectively, with the NRS improving for all replicates. Approximately $90 \%$ of those WGS variants that would be considered FNs relative to the GIAB callset are called in our data, but marked as non-PASSing. MQ is identified as the "culprit" by VQSR in the majority of those

\section{Table 3 Summary of validation experiments performed}

\begin{tabular}{ll}
\hline Experiment & Action \\
\hline $\begin{array}{l}\text { Concordance with SNP Array } \\
\begin{array}{l}\text { Concordance with integrated NGS-derived } \\
\text { variants }\end{array}\end{array}$ & Measure concordance with high-density SNP arrays \\
Measure concordance with Genome In A Bottle (GIAB) NA12878 callset
\end{tabular}

\section{Calling variants from} targeted NGS panel

Intra-run reproducibility

Inter-run reproducibility

Inter-machine reproducibility
Measure concordance with calls from a targeted NGS panel (all calls previously validated either by in-house Sanger assays or the presence of the variant in the sample in Hapmap, 1000G, etc.) Measure concordance for the same sample sequenced in the same run with:

A) Different sample preparations across the same flow cell, or

B) The same sample preparation across different flow cells

Measure concordance for the same sample on the same machine with:

A) The same sample preparation different runs, or

B) Different sample preparation across different runs

Measure concordance for the same sample with the same sample preparation on different machines of the same model in the same run cycle 
Table 4 WES and WGS samples used in different concordance experiments

\begin{tabular}{lll}
\hline Experiment & WES comparison sets & WGS comparison sets \\
\hline Intra-run reproducibility & NA12878: r1-1-1 vs. r1-1-2 & NA12878: r1-1-1 vs. r1-1-3 \\
& NA18507: r2-1-2 vs. r2-1-3 & NA18507: r2-1-3 vs. r2-1-4 \\
\hline Inter-run reproducibility & NA18507: r2-1-2 vs. r3-1-2 & NA18507: r2-1-3 vs. r3-1-3 \\
& NA18507: r2-1-3 vs. r3-1-2 & NA12878: r $r$ 1-1-1-4 vs. r3-1-3 $r$ r-1-1 vs. r3-1-1 \\
& NA12878: r1-1-1 vs. r2-1-1 vs. r3-1-1 & NA12878: r2-2-1 vs. r3-2-1 \\
\hline Inter-machine reproducibility & NA12878: r3-1-1 vs. r3-2-1 & NA12878: r3-1-1 vs. r3-2-1 \\
& NA12878: r4-1-1 vs. r4-2-1 & NA12878: r2-1-1 vs. r2-2-1 \\
\hline Inter-mode reproducibility & NA18507: r4-1-2 vs. r4-2-2 & N/A
\end{tabular}

filtered variants. We note that MQ is no longer a recommended VQSR annotation, a change we would expect to improve VQSR performance.

To investigate the impact of our filtering approach on analytical performance, we plot the precision-recall (PR) curves for WES/WGS SNVs and indels relative to the all and just the high-confidence GIAB callsets in Figure 5. The points show actual PR at the PASS threshold. We observe that the filters are effectively set at the inflection point where relaxing the threshold results more false positives (FPs) than true positives (TPs). We note that for SNVs in the GIAB high-confidence region the rate is

Table 5 Summary of WES coverage statistics

\begin{tabular}{|c|c|c|c|c|c|c|}
\hline Replicate & Mean & $\%$ Bases $>=1 \mathrm{X}$ & $\%$ Bases $>=10 \mathrm{X}$ & $\%$ Bases $>=20 \mathrm{X}$ & $\%$ Bases $>=30 \mathrm{X}$ & $\%$ Coding bases callable \\
\hline \multicolumn{7}{|l|}{ NA12878 } \\
\hline$r 1-1-1$ & 57.4 & 96.8 & 93.6 & 89.2 & 79.3 & 83.8 \\
\hline$r 1-1-2$ & 57.4 & 96.8 & 93.6 & 89.1 & 79.3 & 83.8 \\
\hline$r 2-1-1$ & 66.8 & 96.2 & 92.0 & 85.4 & 75.2 & 79.7 \\
\hline r3-1-1 & 81.8 & 96.7 & 93.6 & 90.7 & 85.1 & 84.8 \\
\hline r3-2-1 & 74.5 & 96.7 & 93.6 & 90.8 & 85.0 & 85.1 \\
\hline $\mathrm{r} 4-1-1$ & 83.7 & 96.7 & 93.7 & 90.9 & 85.5 & 85.0 \\
\hline$r 4-2-1$ & 73.6 & 96.6 & 93.2 & 89.1 & 81.5 & 83.1 \\
\hline \multicolumn{7}{|l|}{ NA12891 } \\
\hline$r 3-1-3$ & 70.9 & 96.5 & 92.4 & 86.0 & 75.8 & 80.1 \\
\hline$r 4-1-3$ & 70.8 & 96.6 & 92.5 & 85.9 & 75.7 & 80.2 \\
\hline$r 4-2-3$ & 60.9 & 96.3 & 91.4 & 82.2 & 69.8 & 76.5 \\
\hline \multicolumn{7}{|l|}{ NA12892 } \\
\hline r3-1-4 & 70.7 & 96.5 & 92.7 & 87.5 & 78.7 & 82.5 \\
\hline$r 4-1-4$ & 71.4 & 96.6 & 92.8 & 87.7 & 79 & 82.7 \\
\hline$r 4-2-4$ & 61.7 & 96.4 & 91.9 & 84.5 & 73.2 & 79.7 \\
\hline \multicolumn{7}{|l|}{ NA18507 } \\
\hline$r 2-1-2$ & 66.4 & 96.4 & 92.1 & 84.7 & 73.8 & 78.7 \\
\hline r2-1-3 & 92.2 & 96.7 & 93.6 & 90.9 & 86.2 & 84.9 \\
\hline r3-1-2 & 81.2 & 96.7 & 93.2 & 88.9 & 81.3 & 82.9 \\
\hline$r 4-1-2$ & 82.8 & 96.7 & 93.3 & 89.2 & 81.7 & 83.2 \\
\hline$r 4-2-2$ & 71.5 & 96.5 & 92.6 & 86.4 & 76.6 & 80.4 \\
\hline \multicolumn{7}{|l|}{ NA10080 } \\
\hline$r 2-1-4$ & 60.7 & 96.4 & 92.1 & 84.5 & 73.2 & 78.4 \\
\hline
\end{tabular}


Table 6 Summary of WGS coverage statistics

\begin{tabular}{|c|c|c|c|c|c|c|}
\hline Replicate & Mean & $\%$ Bases $>=1 \mathrm{X}$ & $\%$ Bases $>=10 \mathrm{X}$ & $\%$ Bases $>=20 \mathrm{X}$ & $\%$ Bases $>=30 \mathrm{X}$ & $\%$ Coding bases callable \\
\hline \multicolumn{7}{|l|}{ NA12878 } \\
\hline$r 1-1-1$ & 51.0 & 95.4 & 94.9 & 94.3 & 93.1 & 91.8 \\
\hline$r 1-1-3$ & 49.8 & 95.4 & 94.9 & 94.3 & 93.1 & 91.9 \\
\hline$r 2-1-1$ & 36.5 & 95.4 & 94.7 & 93.5 & 86.9 & 91.6 \\
\hline$r 2-2-1$ & 34.6 & 95.4 & 94.7 & 93.2 & 82.9 & 91.2 \\
\hline r3-1-1 & 49.9 & 95.5 & 95.0 & 94.5 & 93.5 & 91.9 \\
\hline r3-2-1 & 39.8 & 95.5 & 94.8 & 93.9 & 89.4 & 91.0 \\
\hline \multicolumn{7}{|l|}{ NA12891 } \\
\hline r3-1-2 & 42.7 & 96.8 & 96.1 & 94.3 & 88.7 & 91.2 \\
\hline \multicolumn{7}{|l|}{ NA12892 } \\
\hline$r 3-1-4$ & 45.1 & 95.2 & 90.8 & 86.6 & 81.3 & 90.9 \\
\hline \multicolumn{7}{|l|}{ NA18507 } \\
\hline$r 2-1-3$ & 30.0 & 96.8 & 95.7 & 89.4 & 65.8 & 87.3 \\
\hline$r 2-1-4$ & 35.8 & 96.8 & 96.0 & 92.3 & 83.4 & 90.2 \\
\hline r3-1-3 & 51.7 & 96.9 & 96.3 & 95.4 & 91.6 & 91.6 \\
\hline \multicolumn{7}{|l|}{ NA10080 } \\
\hline$r 2-1-2$ & 35.1 & 96.9 & 96.1 & 92.0 & 81.7 & 89 \\
\hline
\end{tabular}

Percent RefSeq Coding Bases Callable reports the percentage of all RefSeq coding exons bases (as downloaded from the UCSC Genome Browser) considered callable.

approximately 1:1, i.e. roughly equal numbers of FPs and TPs result from relaxed filtering. In situations, such as clinical workflows, where FNs are very problematic and FPs less so, treating all variants in the high-confidence region as PASSing might be a good tradeoff.

\section{Targeted gene panel}

Table 7 lists the site-level sensitivity (NRS), specificity and genotype-level NRC relative to Sanger validated and or HapMap, $1000 \mathrm{G}$ and NIST genotypes in 129 kilobases (kb) of target from a clinical multigene panel (the ASD Panel callset). Not all variants were validated during panel design and testing, e.g. intronic variants or the Sanger results are inconclusive so we cannot always determine if a WES variant is a false positive (FP). Thus we report a mix of FPs and "excess" positives, variants discovered with NGS that were not Sanger confirmed or not conclusively reported elsewhere.

Table 8 lists the false negative (FN) variants for NA12878 in the panel's target regions. The most common FN, 3/6 WES FNs and 9/11 WGS FNs, is a single-base deletion in

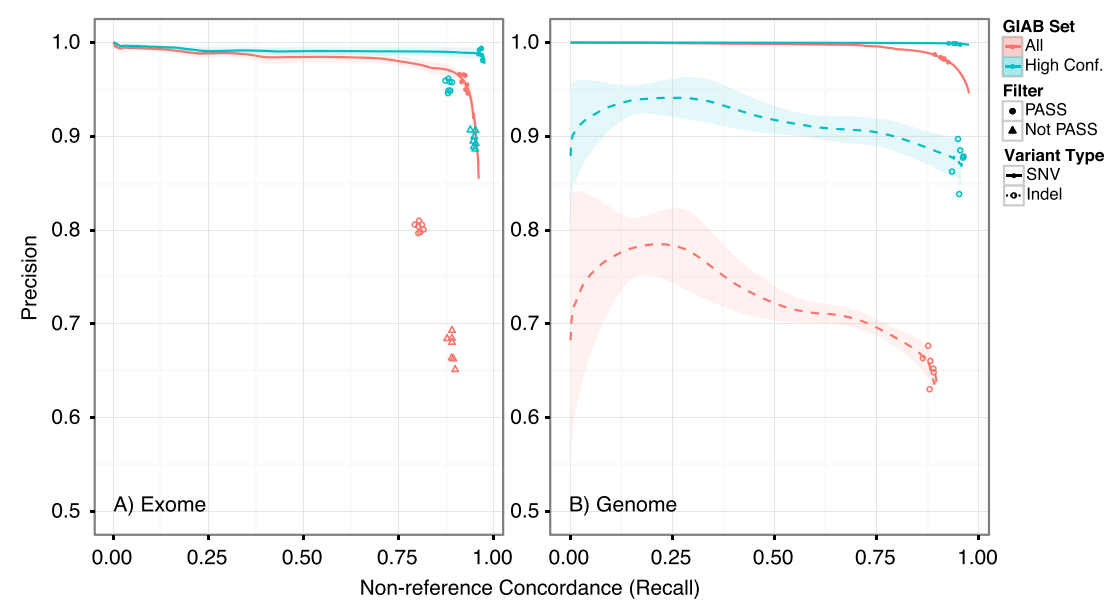

Figure 5 Precision vs. non-reference concordance (NRC) for WES (A) and WGS (B) SNVs (solid line) and Indels (dashed line) as a function of VQSR VQSLoD score relative to GIAB high confidence and all variant sets. Thick line is mean across all replicates; shaded region shows the standard deviation. Points show the PR at the VQSR PASSing threshold. VQSR is not applied in WES indels; points show PR for PASSing and all variants (both PASS and not-PASS). 
Table 7 Site-level sensitivity (NRS), specificity and genotype-level NRC relative to Sanger validated and or Hapmap or 1000G-reported variants in $129 \mathrm{~Kb}$ of target from a clinical gene panel (the ASD panel callset)

\begin{tabular}{|c|c|c|c|c|c|c|c|c|}
\hline Replicate & Variants & TP & FP/EP & $\mathrm{FN}$ & TN & Diff. Alleles & Sensitivity (NRS) & NRC \\
\hline \multicolumn{9}{|c|}{ NA12878 Exome } \\
\hline$r 1-1-1$ & 48 & 40 & 5 & 3 & 0 & 0 & $93.0 \%$ & $81.4 \%$ \\
\hline$r 1-1-2$ & 46 & 40 & 2 & 3 & 1 & 0 & $93.0 \%$ & $83.7 \%$ \\
\hline r2-1-1 & 45 & 37 & 1 & 6 & 1 & 0 & $86.0 \%$ & $74.4 \%$ \\
\hline r3-1-1 & 44 & 40 & 0 & 3 & 1 & 0 & $93.0 \%$ & $83.7 \%$ \\
\hline r3-2-1 & 44 & 40 & 0 & 3 & 1 & 0 & $93.0 \%$ & $83.7 \%$ \\
\hline$r 4-1-1$ & 44 & 39 & 0 & 4 & 1 & 0 & $90.7 \%$ & $79.1 \%$ \\
\hline$r 4-2-1$ & 44 & 39 & 0 & 4 & 1 & 0 & $90.7 \%$ & $81.4 \%$ \\
\hline \multicolumn{9}{|c|}{ NA12878 Genome } \\
\hline$r 1-1-1$ & 64 & 54 & 1 & 8 & 1 & 1 & $87.3 \%$ & $81.0 \%$ \\
\hline$r 1-1-3$ & 74 & 53 & 11 & 9 & 1 & 1 & $85.7 \%$ & $79.4 \%$ \\
\hline r2-1-1 & 73 & 53 & 10 & 9 & 1 & 1 & $85.7 \%$ & $77.8 \%$ \\
\hline r2-2-1 & 73 & 54 & 10 & 8 & 1 & 1 & $87.3 \%$ & $81.0 \%$ \\
\hline r3-1-1 & 71 & 55 & 8 & 7 & 1 & 1 & $88.9 \%$ & $81.0 \%$ \\
\hline r3-2-1 & 64 & 52 & 1 & 10 & 1 & 1 & $84.1 \%$ & $74.6 \%$ \\
\hline
\end{tabular}

Not all variants in this interval were validated, e.g. intronic variants, so we cannot conclusively determine if a variant is a false positive. Instead we report "excess positives", variants discovered in the NGS replicates that were not Sanger confirmed or conclusively reported elsewhere.

Table 8 False negative variants in NA12878 relative to the ASD panel callset

\begin{tabular}{|c|c|c|c|c|c|}
\hline Position & Variant & Truth genotype & Called in & $\begin{array}{l}\text { Would be } \\
\text { reviewed }\end{array}$ & Note \\
\hline \multicolumn{6}{|l|}{ Exome } \\
\hline chr10:89720907 & $\mathrm{T}>\mathrm{G}$ & $\mathrm{T} / \mathrm{G}$ & $4 / 7$ & No & Called, but filtered in some replicates for MQ \\
\hline chr12:2614070 & $\mathrm{G}>\mathrm{T}$ & $\mathrm{G} / \mathrm{T}$ & $6 / 7$ & Yes (silent) & Called, but filtered in one replicate \\
\hline chrX:15863648 & $G A>G$ & $\mathrm{GA} / \mathrm{G}$ & $0 / 7$ & No & Not called in any replicate; 10 bp homopolymer \\
\hline chrX:135115669 & $G A>G$ & $\mathrm{GA} / \mathrm{G}$ & $0 / 7$ & No & Called, but filtered in two replicates; 11 bp homopolymer \\
\hline chrX:152954025 & $A>G$ & $\mathrm{G} / \mathrm{G}$ & $6 / 7$ & Yes (UTR) & Low depth region \\
\hline chrX:153287314 & $\mathrm{TG}>\mathrm{T}$ & $\mathrm{TG} / \mathrm{T}$ & $0 / 7$ & Yes (UTR) & Called, but filtered in all replicates for QD; 10 bp homopolymer \\
\hline \multicolumn{6}{|l|}{ Genome } \\
\hline chr5:176639217 & $\mathrm{TA}>\mathrm{T}$ & $\mathrm{TA} / \mathrm{T}$ & $0 / 6$ & No & Not called in any replicate; 13 bp homopolymer \\
\hline chr7:146805220 & $A T>A$ & AT/A & $0 / 6$ & No & Not called in any replicate; 11 bp homopolymer \\
\hline chr10:89720633 & $C T>C$ & $\mathrm{CT} / \mathrm{C}$ & $0 / 6$ & No & Not called in any replicate; 15 bp homopolymer \\
\hline chr10:89720907 & $\mathrm{T}>\mathrm{G}$ & $\mathrm{T} / \mathrm{G}$ & $0 / 6$ & No & Called, but filtered in all replicates \\
\hline chr11:70348852 & $\mathrm{G}>C G$ & CG/CG & $0 / 6$ & No & Called, but as heterozygous in all replicates; inside 12 bp homopolymer \\
\hline chrX:15863648 & $\mathrm{GA}>\mathrm{G}$ & GA/G & $0 / 6$ & No & Not called in any replicate; 10 bp homopolymer \\
\hline chrX:132888207 & $\mathrm{TA}>\mathrm{T}$ & $\mathrm{TA} / \mathrm{T}$ & $3 / 6$ & No & Not called in three replicates; 16 bp homopolymer \\
\hline chrX:135067675 & $\mathrm{G}>\mathrm{C}$ & $\mathrm{G} / \mathrm{C}$ & $5 / 6$ & Yes (missense) & Not called in one replicate \\
\hline chrX:135115669 & $G A>G$ & GA/G & $1 / 6$ & No & Called in one replicate; 11 bp homopolymer \\
\hline chrX:153287314 & $\mathrm{TG}>\mathrm{T}$ & $\mathrm{TG} / \mathrm{T}$ & $1 / 6$ & Yes (UTR) & Called in one replicate; $10 \mathrm{bp}$ homopolymer \\
\hline chrX:153357614 & $\mathrm{TA}>\mathrm{T}$ & $\mathrm{TA} / \mathrm{T}$ & $1 / 6$ & No & Called in one replicate; 13 bp homopolymer \\
\hline
\end{tabular}

Most common error mode is single base deletions in homopolymer regions. Each variant is annotated as to whether it would be further reviewed in interpretations workflow (Yes) or automatically filtered out from further consideration (No). 
a homopolymer region, e.g. a poly-A tract. The sequencing instrument's error rate increases in homopolymer regions, making it more difficult to detect indels in these contexts $[19,20]$. As such, these FNs are not unexpected. For these particular variants, the deletion is typically detected in a small fraction of the reads $(<10 \%)$, too few to accurately call the heterozygous deletion. A pileup for a representative example is included in the Additional file 1. Increasing the read depth does reduce the number FNs that result from variation in filtering, but not the missed deletions. Most of the single-base deletions are also not reliably called in high-depth (over 250-fold) targeted sequencing. As in the WES/WGS, the deletions are detected in only a small fraction of the reads.

The FN variants in Table 8 impact the analytical sensitivity, but may not necessarily impact clinical sensitivity. The variants chrX:15863648GA $>$ G and chrX:135115669GA $>$ G, for example, occur in homopolymer intronic regions outside the invariant splice site, and thus are unlikely to result in a change in protein functionality and would not be considered for in-depth review. We annotated each variant in Table 8 as to whether it would be reviewed in a variant interpretation workflow focused exclusively on known disease mutations or exonic/splice variants; $50 \%$ or more variants would be filtered out automatically and not reviewed further.

To evaluate our sensitivity, more generally, for the subset of variants more likely to be pathogenic, we tested the sensitivity relative to the high-confidence lossof-function (LoF) mutations in NA12878 reported in MacArthur et al.'s study of LoF variants in the 1000 Genomes cohort [21]. We detected 56-57 of 61 variants in the WES replicates (sensitivity of $92-93 \%$ ) and $68-70$ of 73 variants in the WGS replicates (sensitivity of 93-96\%). As with other NGS-derived callsets, directly extrapolating sensitivity from this dataset is likely an overestimate (because the dataset may not include variant types/regions that are difficult to sequence).

In those NA12878 replicates with multiple FPs relative to the ASD panel callset, the majority are artifacts created by two larger structural variants in the introns of SHANK3 detected manually during the review of discordant calls. Although the individual variants are PASSing, manual review of the pileup (Additional file 1) immediately shows these variants to be artifacts of the larger variant. The same variant caller can produce very different calls for different sequencing replicates, even with similar coverage, in and around these structural variants and repeat regions. The differences are not in the filtering, but in which variants the caller emits. These artifactual variants manifest as FPs, and will reduce the NRS and NRC, but not the concordance, when using NGS datasets as truth. The remaining FPs are non-recurrent errors in 1-2 replicates.

\section{Technical replicates}

Figure 6 shows the concordance, NRS and NRC for all pairwise comparisons of NA12878 and NA18507 replicates, marked as the kind of comparison, i.e. intra-run, inter-run, inter-machine, inter-mode, inter-library, where appropriate. Many comparisons are of more than one kind; those without a clear primary specific classification are marked as "other". Visual inspection does not indicate any clear relationship between comparison kind and concordance. To quantitatively assess the contribution of these different kinds of comparisons to the concordance, we performed a multiple linear regression analysis

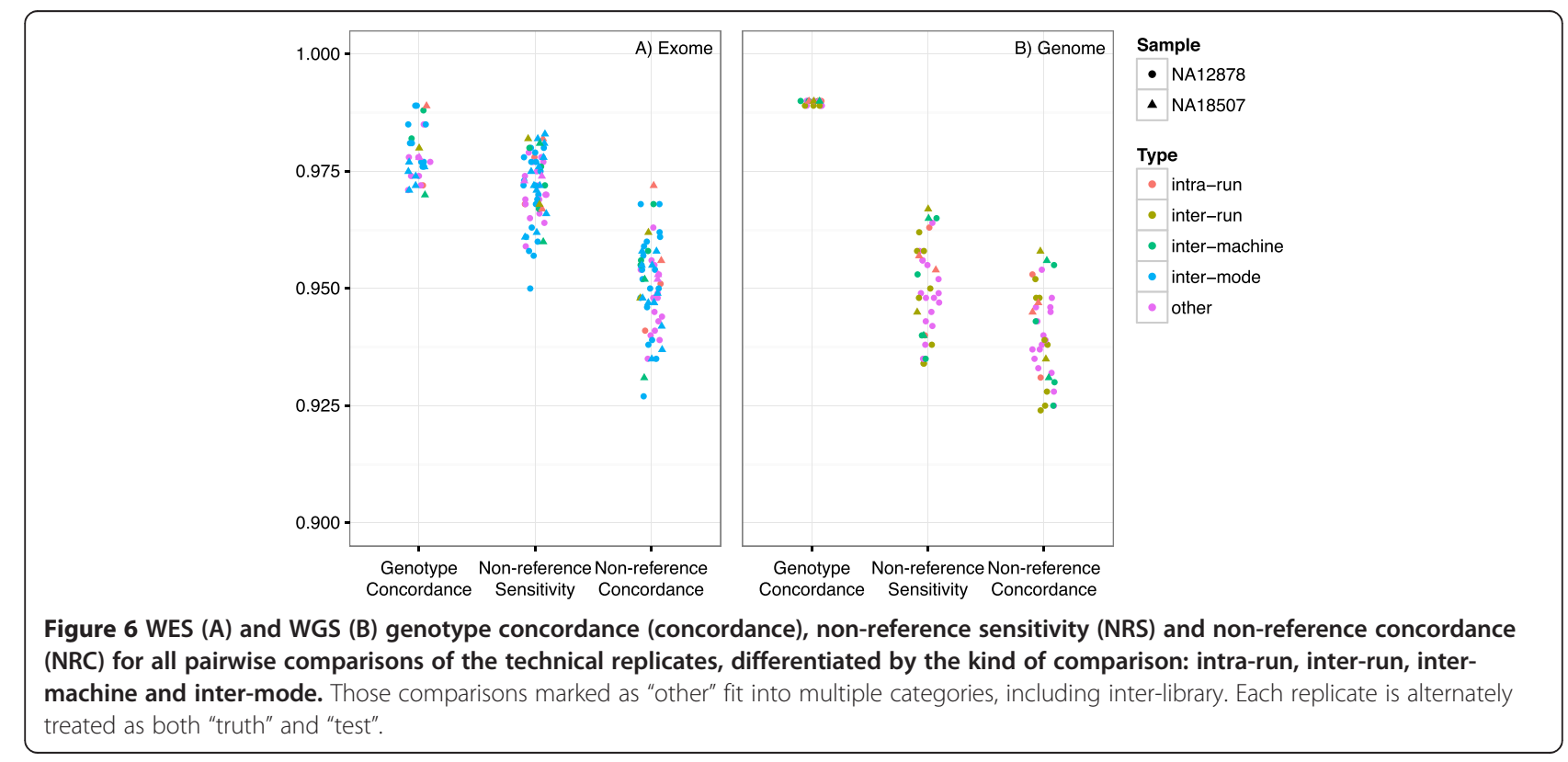


of the five different comparison kinds as binary variables and the sample ID as a binary control variable on concordance for all NA12878 and NA18507 replicate pairs. A separate regression analysis was performed for WES (31 pairs) and WGS (18 pairs). WES did not significantly differ from the null model (sample covariate alone) at a threshold of 0.05 , while WGS did significantly differ (P $=0.016)$. We further analyzed each WGS comparison type individually. The only comparison kind to be reported as significant $(\mathrm{P}=.0044)$ at a threshold of 0.0125 (Bonferroni corrected for 4 tests) was WGS inter-library; the coefficient estimate translates to a reduction in concordance of 0.0006 , i.e. from $99 \%$ to $98.94 \%$. The regression analysis is described in more detail in the Additional file 1.

Table 9 lists the fractions of different types of variants uniformly called in different fractions of replicates. Of all variants identified in WES data from the NA12878 reference sample $74 \%$ of indels and $89 \%$ of SNVs were called PASSing or not PASSing in all seven replicates. In NA12878 WGS 52\% of indels and $88 \%$ of SNVs were uniformly called in all six replicates. The non-uniformity is only partially a result of variation in depth of coverage. Restricting the analysis to the "squared-off" intersection of confidently callable regions across all replicates increases the percentage of SNVs in all replicates by approximately 2-4 and indels by $7-10$. Further restricting the analysis to the GIAB high-confidence regions further increases the percentages of variants uniformly called across all replicates by a similar amount: $89.5 \%$ of indels and $97.9 \%$ of SNVs are uniformly called in the NA12782 WES replicates, and $71.0 \%$ of indels and $93.2 \%$ of SNVs in the WGS replicates. The number of uniquely observed variants, i.e. variants called in only one replicate, is in the range [118-252] and [29395-54746] for NA12878

\begin{tabular}{|c|c|c|c|c|c|c|c|}
\hline NA12878 WES & $1 / 7$ & $2 / 7$ & $3 / 7$ & $4 / 7$ & $5 / 7$ & $6 / 7$ & $7 / 7$ \\
\hline SNV & 3.20 & 1.37 & 1.18 & 1.28 & 1.42 & 2.69 & 88.85 \\
\hline Indel & 10.25 & 4.60 & 2.62 & 2.33 & 2.51 & 3.71 & 73.99 \\
\hline NA18507 WES & $1 / 5$ & $2 / 5$ & $3 / 5$ & $4 / 5$ & $5 / 5$ & & \\
\hline SNV & 2.87 & 1.60 & 1.47 & 2.62 & 91.43 & & \\
\hline Indel & 7.61 & 3.57 & 2.96 & 3.70 & 82.17 & & \\
\hline NA12878 WGS & $1 / 6$ & $2 / 6$ & $3 / 6$ & $4 / 6$ & $5 / 6$ & $6 / 6$ & \\
\hline SNV & 3.40 & 1.76 & 1.52 & 1.79 & 3.69 & 87.80 & \\
\hline Indel & 20.20 & 9.89 & 6.11 & 5.17 & 7.07 & 51.60 & \\
\hline NA18507 WGS & $1 / 3$ & $2 / 3$ & $3 / 3$ & & & & \\
\hline SNV & 2.61 & 2.93 & 94.46 & & & & \\
\hline Indel & 20.01 & 13.10 & 66.89 & & & & \\
\hline
\end{tabular}

WES and WGS, respectively, and [182-335] and [66342-104224] for NA18507 WES and WGS respectively.

\section{Conclusions}

We evaluated the intra-run, inter-run, inter-mode and inter-machine reproducibility, concordance with orthogonal technologies (microarray, Sanger) and sensitivity relative to known variant sets (GIAB, MacArthur et al.) for both WES and WGS across multiple technical replicates of five different reference samples. These analyses were performed as part of the validation of WES/WGS as a clinical test in our laboratory and as a result focus on a single informatics pipeline and exome capture technology. However since both technologies are widely used or are generally reflective of alternative approaches, the results presented here should be broadly relevant to anyone interested in the performance characteristics of WES/WGS.

The state of reference callsets is changing rapidly, with GIAB and GeT-RM being just two examples of newly available callsets for NA12878. Those and similar resources will continue to improve and expand, and therefore any analysis using such resources represents a snapshot of a particular moment in time. The different sensitivities observed for different kinds of callsets (microarray, NGS-derived datasets like GIAB and Sanger sequencing) shows the continued challenges in genotyping different kinds of variation as well as the continuing challenges in building high-quality, comprehensive reference callsets The comparisons with Sanger data, for instance, show that indels in homopolymer regions are under-detected, while the comparisons to NGS-derived datasets, like GIAB, show how the structural variants and repeat regions can be interpreted very differently by different variant callers, even when restricting to highcoverage, high-confidence regions.

At the time of this report, there are no absolute standards for the analytical performance characteristics for WES/WGS but the results presented here fall within the ACMG suggested ranges [8]. By the nature of WES/ WGS, most of the reported analyses are focused on analytical performance characteristics that are independent of any particular clinical scenario. We have successfully used the documented workflow, however, to make a molecular diagnosis in 4 of 8 pilot clinical WES cases, indicating that its performance translates to a useful clinical sensitivity that is on par with that reported by other laboratories [22] and at the suggested 50\% threshold for cost effectiveness [23]. The difference between the clinical sensitivity and the much higher analytical sensitivity for SNVs and small indels reported here reinforces the need to continue to improve our sequencing, variant calling and variant interpretation technologies (even a $1 \%$ false negative rate in WES/WGS translates into many 
variants in absolute terms) and our ability to identify other variant types, e.g. CNVs, which contribute to disease burden.

\section{Additional file}

Additional file 1: Supplemental methods, figures and tables.

\section{Abbreviations \\ SNV: Single nucleotide variant; WES: Whole exome sequencing; WGS: Whole genome sequencing; GAP: Genome analysis pipeline; RM: Reference material; Concordance: Genotype concordance; NRS: Non-reference sensitivity; NRC: Non-reference concordance; GIAB: Genome in a bottle.}

\section{Competing interests}

The Icahn School of Medicine at Mount Sinai offers fee-for-service clinical whole exome sequencing and genomic sequencing services. The authors declare to no other competing interests.

\section{Authors' contributions}

MDL implemented the genome pipeline, designed and performed the analyses and drafted the manuscript. TB designed the analysis and performed the Sanger validation of the targeted regions. HS performed the primary analysis of the sequencing data. LE, RK and TB designed the number and types of sequencing runs and supervised the clinical aspects of the validation. MM, OJ, and YK supervised and performed the sequencing. EES and AK supervised the sequencing and pipeline development/deployment. All authors read and approved the final manuscript.

\section{Acknowledgements}

The authors wish to thank Douglas Ruderfer, Menachem Fromer, David Mittelman and the reviewers for their many helpful comments on this manuscript. This work was supported in part through the computational resources and staff expertise provided by the Department of Scientific Computing at the Icahn School of Medicine at Mount Sinai.

Received: 8 January 2014 Accepted: 10 April 2014

Published: 23 April 2014

\section{References}

1. Gargis AS, Kalman L, Berry MW, Bick DP, Dimmock DP, Hambuch T, Lu F, Lyon E, Voelkerding KV, Zehnbauer BA, Agarwala R, Bennett SF, Chen B, Chin ELH, Compton JG, Das S, Farkas DH, Ferber MJ, Funke BH, Furtado MR, Ganova-Raeva LM, Geigenmüller U, Gunselman SJ, Hegde MR, Johnson PLF, Kasarskis A, Kulkarni S, Lenk T, Liu CSJ, Manion M, et al: Assuring the quality of next-generation sequencing in clinical laboratory practice. Nat Biotechnol 2012, 30:1033-1036.

2. Rieber N, Zapatka M, Lasitschka B, Jones D, Northcott P, Hutter B, Jäger N, Kool M, Taylor M, Lichter P, Pfister S, Wolf S, Brors B, Eils R: Coverage bias and sensitivity of variant calling for four whole-genome sequencing technologies. PLoS One 2013, 8:e66621.

3. Lam HYK, Clark MJ, Chen R, Chen R, Natsoulis G, O'Huallachain M, Dewey FE, Habegger L, Ashley EA, Gerstein MB, Butte AJ, Ji HP, Snyder M: Performance comparison of whole-genome sequencing platforms. Nat Biotechnol 2012, 30:78-82.

4. Asan, Xu Y, Jiang H, Tyler-Smith C, Xue $Y$, Jiang $T$, Wang J, Wu M, Liu X, Tian G, Wang J, Wang J, Yang $H$, Zhang $X$ : Comprehensive comparison of three commercial human whole-exome capture platforms. Genome Biol 2011, 12:R95.

5. Clark MJ, Chen R, Lam HYK, Karczewski KJ, Chen R, Euskirchen G, Butte AJ, Snyder M: Performance comparison of exome DNA sequencing technologies. Nat Biotechnol 2011, 29:908-914.

6. O'Rawe J, Jiang T, Sun G, Wu Y, Wang W, Hu J, Bodily P, Tian L, Hakonarson H, Johnson WE, Wei Z, Wang K, Lyon GJ: Low concordance of multiple variant-calling pipelines: practical implications for exome and genome sequencing. Genome Med 2013, 5:28.

7. Liu X, Han S, Wang Z, Gelernter J, Yang B-Z: Variant callers for next-generation sequencing data: a comparison study. PLoS One 2013, 8:e75619.
8. Rehm HL, Bale SJ, Bayrak-Toydemir P, Berg JS, Brown KK, Deignan JL, Friez MJ, Funke BH, Hegde MR, Lyon E: ACMG clinical laboratory standards for next-generation sequencing. Genet Med 2013, 15:733-747.

9. DePristo MA, Banks E, Poplin R, Garimella KV, Maguire JR, Hartl C, Philippakis AA, del Angel G, Rivas MA, Hanna M, McKenna A, Fennell TJ, Kernytsky AM, Sivachenko AY, Cibulskis K, Gabriel SB, Altshuler D, Daly MJ: A framework for variation discovery and genotyping using next-generation DNA sequencing data. Nat Genet 2011, 43:491-498.

10. McKenna A, Hanna M, Banks E, Sivachenko A, Cibulskis K, Kernytsky A, Garimella K, Altshuler D, Gabriel S, Daly M, DePristo MA: The genome analysis toolkit: a MapReduce framework for analyzing next-generation DNA sequencing data. Genome Res 2010, 20:1297-1303.

11. Zook JM, Salit M: Genomes in a bottle: creating standard reference materials for genomic variation - why, what and how? Genome Biol 2011, 12(Suppl 1):31.

12. Zook JM, Chapman B, Wang J, Mittelman D, Hofmann O, Hide W, Salit M: Integrating human sequence data sets provides a resource of benchmark SNP and indel genotype calls. Nat Biotechnol 2014, 32:246-251.

13. Li H, Durbin R: Fast and accurate short read alignment with Burrows-Wheeler transform. Bioinformatics 2009, 25:1754-1760.

14. Picard Team: Picard. In 2012.

15. Cingolani P, Platts A, Wang LL, Coon M, Nguyen T, Wang L, Land SJ, Lu X, Ruden DM: A program for annotating and predicting the effects of single nucleotide polymorphisms, SnpEff: SNPs in the genome of Drosophila melanogaster strain w1118; iso-2; iso-3. Fly (Austin) 2012, 6:80-92.

16. Quinlan AR, Hall IM: BEDTools: a flexible suite of utilities for comparing genomic features. Bioinformatics 2010, 26:841-842.

17. Shen R, Fan J-B, Campbell D, Chang W, Chen J, Doucet D, Yeakley J, Bibikova M, Wickham Garcia E, McBride C, Steemers F, Garcia F, Kermani BG, Gunderson K, Oliphant A: High-throughput SNP genotyping on universal bead arrays. Mutat Res 2005, 573:70-82.

18. Abecasis GR, Auton A, Brooks LD, DePristo MA, Durbin RM, Handsaker RE, Kang HM, Marth GT, McVean GA: An integrated map of genetic variation from 1,092 human genomes. Nature 2012, 491:56-65.

19. Albers CA, Lunter G, MacArthur DG, McVean G, Ouwehand WH, Durbin R: Dindel: accurate indel calls from short-read data. Genome Res 2011, 21:961-973.

20. Highnam G, Franck C, Martin A, Stephens C, Puthige A, Mittelman D: Accurate human microsatellite genotypes from high-throughput resequencing data using informed error profiles. Nucleic Acids Res 2013, 41:e32.

21. MacArthur DG, Balasubramanian S, Frankish A, Huang N, Morris J, Walter K, Jostins L, Habegger L, Pickrell JK, Montgomery SB, Albers CA, Zhang ZD, Conrad DF, Lunter G, Zheng H, Ayub Q, DePristo MA, Banks E, Hu M, Handsaker RE, Rosenfeld JA, Fromer M, Jin M, Mu XJ, Khurana E, Ye K, Kay M, Saunders Gl, Suner M-M, Hunt T, et al: A systematic survey of loss-of-function variants in human protein-coding genes. Science 2012, 335:823-828.

22. Yang Y, Muzny DM, Reid JG, Bainbridge MN, Willis A, Ward PA, Braxton A, Beuten J, Xia F, Niu Z, Hardison M, Person R, Bekheirnia MR, Leduc MS, Kirby A, Pham P, Scull J, Wang M, Ding Y, Plon SE, Lupski JR, Beaudet AL, Gibbs RA, Eng CM: Clinical whole-exome sequencing for the diagnosis of Mendelian disorders. N Engl J Med 2013, 369:1502-1511.

23. Shashi V, McConkie-Rosell A, Rosell B, Schoch K, Vellore K, McDonald M, Jiang $Y-H$, Xie P, Need A, Goldstein DG: The utility of the traditional medical genetics diagnostic evaluation in the context of next-generation sequencing for undiagnosed genetic disorders. Genet Med 2013, 16:176-182

doi:10.1186/1755-8794-7-20

Cite this article as: Linderman et al:: Analytical validation of whole exome and whole genome sequencing for clinical applications. BMC Medical Genomics 2014 7:20. 